Invited Paper

\title{
Fabrication and Characterization of
}

\section{Carbon Nanotube Field-Effect Transistor Biosensors}

\author{
Matthew R. Leyden, Canan Schuman, Tal Sharf, Josh Kevek, Vincent T. Remcho, Ethan D. Minot \\ Dept. of Physics, Oregon State University, Corvallis, OR, USA 97331;
}

\begin{abstract}
We report the fabrication of carbon nanotube field-effect transistors for biosensing applications and the development of protocols for reliable protein and DNA sensing. The sensing method we employ is 'label free', relying only on the intrinsic charge of the biological molecule of interest. We discuss fabrication issues that we have solved, for example the preparation of clean surfaces, and the sensing protocol issues that we have encountered. Polylysine and ss-dna, highly charged biological molecules, are used as model systems to illustrate the performance of our biosensors.
\end{abstract}

Keywords: Label-free biosensor, Biosensor, Carbon nanotube, Nanoelectronics, Protein sensor, Mass-transport, Microfluidics.

\section{INTRODUCTION}

Over the last decade, a variety of protein and DNA sensors have been developed that circumvent the need for fluorescent labeling and fluorescence imaging. ${ }^{1-6}$ Development of these label-free detection schemes is motivated by the need for faster, lower-cost detection of biomolecules. By removing the need for labeling and imaging, researchers hope to make biosensors more accessible to doctors and patients. This access to quantitative information about the presence of specific biomolecules in a patient's body is a critical step towards more accurate and personalized medical care. ${ }^{7}$

To detect unlabeled biomolecules, label-free detection schemes utilize intrinsic protein properties such as polarizability, mass and electric charge. Surface plasmon resonance, ${ }^{1}$ optical waveguides ${ }^{2}$ and impedance sensors ${ }^{3}$ rely on the dielectric constant of biomolecules. The mass of biomolecules creates a signal for sensors based on micromechanical oscillators. ${ }^{4}$ Charged biomolecules can be detected by nanowire field effect transistors (FETs) ${ }^{5}$ and carbon nanotube (CNT) FETs. ${ }^{6}$ In the class of charge-sensitive biosensors, semiconducting CNTs are extremely promising. Carbon nanotubes are hollow cylinders of $\mathrm{sp}^{2}$ bonded carbon with a typical diameter of 1-2 $\mathrm{nm}$. All atoms of the CNT are on the surface, carrier mobility is exceptionally high, and the $\mathrm{sp}^{2}$ bonded carbon lattice is extremely stable in biological environments.

Figure 1 illustrates the basic transduction mechanism of a CNT FET biosensor. The conductance $G$ of the semiconducting CNT can be tuned either by ions in the liquid environment (Fig. 1a) or, equivalently, charged proteins binding to the CNT surface (Fig. 1b). Free carriers in the CNT are capacitively coupled to charges in the environment, therefore, the number of free carriers is extremely sensitive to charges in the environment. Conductance of the semiconducting CNT is proportional to the number of free carriers. Protein binding can have additional effects on the conductance of a CNT FET device. These additional effects have been the subject of detailed investigations. ${ }^{8}$

Organic Semiconductors in Sensors and Bioelectronics III, edited by Ruth Shinar, loannis Kymissis, Proc. of SPIE Vol. $7779,77790 \mathrm{H} \cdot$ (C) 2010 SPIE · CCC code: 0277-786X/10/\$18 - doi: 10.1117/12.861329 

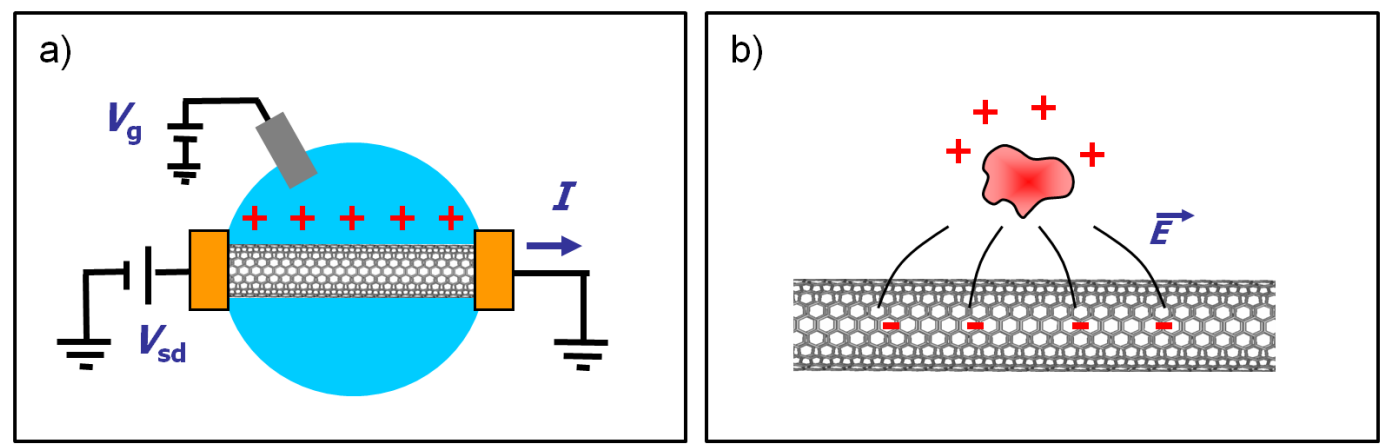

Figure 1. Gating of CNT field effect transistors. a) A semiconducting CNT is contacted by two metal electrodes (yellow). The gate voltage $V_{\mathrm{g}}$ sets the electrostatic potential of the buffer solution (blue). The gate voltage is applied using a $\mathrm{Ag} / \mathrm{AgCl}$ reference electrode. Ions that condense on the CNT surface (shown as + symbols) induce carriers of the opposite charge in the CNT. b) A positively charge protein will also induce carriers of the opposite in the CNT.

Figure 2 shows data from a p-type CNT-FET biosensor. The free-carriers in the CNT are holes; the free-carriers are repelled by positive ions or positive proteins. Depletion of free carriers causes a decrease in the conductance of the sensor. In Figure 2a, conductance is tuned by changing the electrostatic potential $V_{\mathrm{g}}$ of the electrolyte solution that surrounds the sensor. In Figure $2 b$, the conductance changes due to proteins binding on the surface of the sensor. The total conductance change caused by protein binding can be expressed as an equivalent change in $V_{\mathrm{g}}$. In Fig $2 \mathrm{~b}$, the binding of polylysine is equivalent to a shift $\Delta V_{\mathrm{g}}=+55 \mathrm{mV}$.
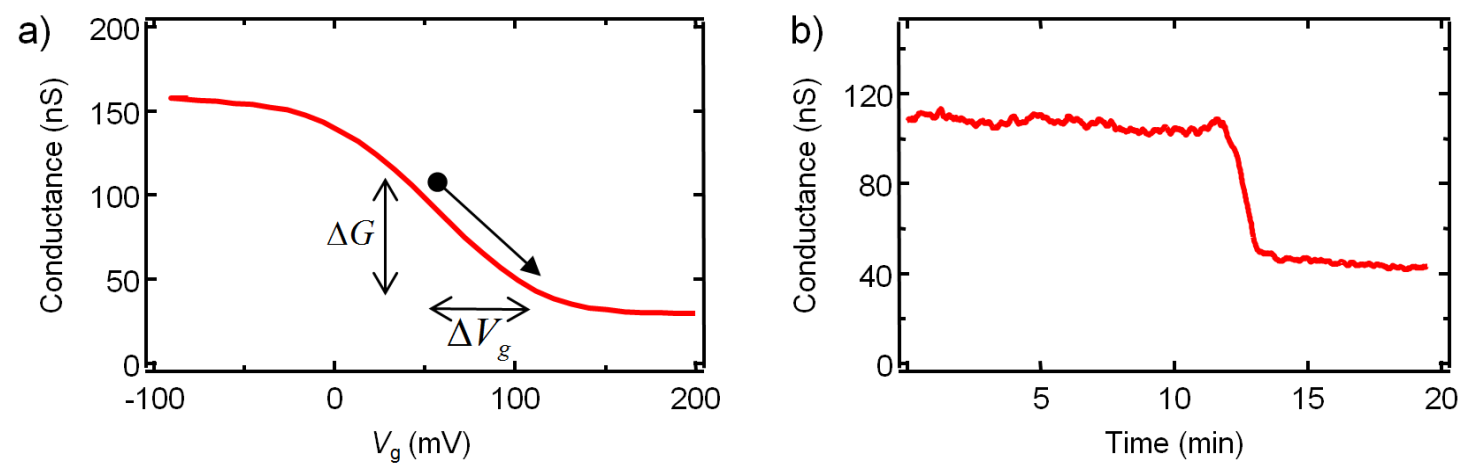

Figure 2. Measurements from a p-type CNT-FET biosensor. a) Transistor curve (conductance vs. $V_{\mathrm{g}}$ ) of sensor gated by an electrolyte solution. $\Delta G$ and $\Delta V_{\mathrm{g}}$ illustrate the effect of polylysine absorption. b) Real time sensing data with $V_{\mathrm{g}}$ set to 50 $\mathrm{mV}$. Polylysine (MW110kD) $10 \mu \mathrm{g} / \mathrm{ml}$ in phosphate buffered saline is injected at 12 minutes. A flow rate of $3 \mathrm{ml} / \mathrm{hr}$ is maintained throughout the experiment.

While CNT FETs are among the most promising devices for label-free biosensing, researchers continue to wrestle with issues such as unknown noise sources, manufacturing reliability, spurious signals, and slow detection speed. In this contribution we describe the methods that we have developed for device manufacturing and controlling the sensing environment to avoid spurious signals. Using these methods, we have characterized detection speed and confirmed theoretical predictions of protein mass-transport.

\section{CIRCUIT FABRICATION}

\subsection{Overview}

Many fabrication processes have been developed for nanotube transistors. ${ }^{9-11}$ These transistor devices can be broadly classified as (i) individual CNT devices and (ii) CNT network devices. Individual CNTs circuits are commonly 
fabricated by patterning nanotube catalyst onto the device substrate and then growing CNTs directly on the device using catalyzed chemical vapor deposition (CVD). ${ }^{9}$ This method relies on Poissonian statistics to randomly produce devices with zero, one or two CNTs (only a fraction of devices will have an individual CNT). Nanotube network devices typically involve fewer fabrication steps and higher yields of working devices. ${ }^{10,11}$ However, these network devices do not give researchers insight into transduction mechanisms at the single CNT level. Moreover, individual CNT sensors are more promising than network sensors for single molecule sensing applications.
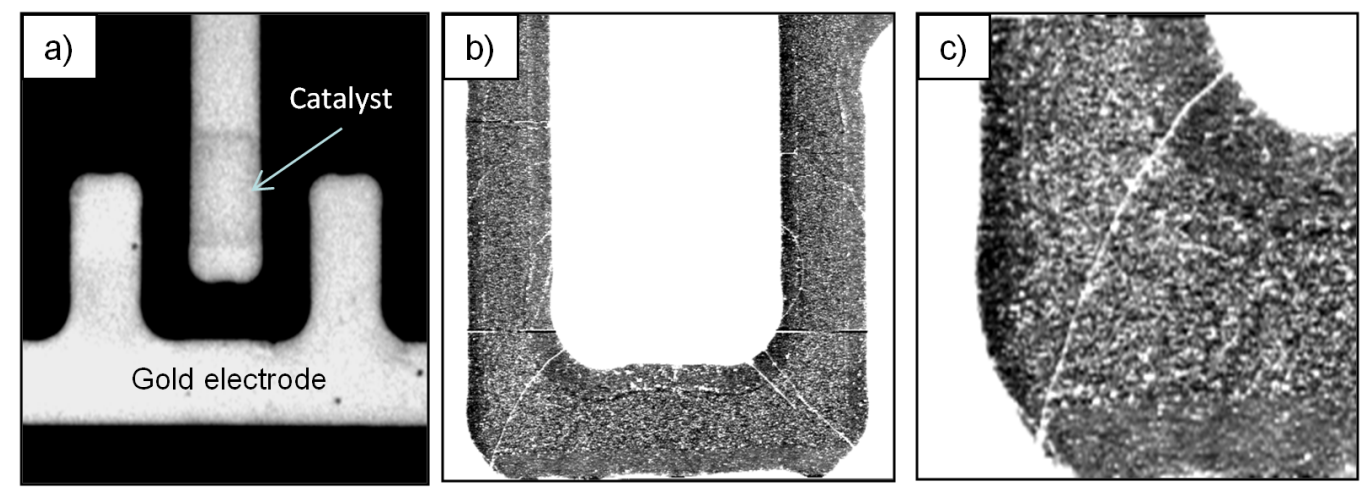

Figure 3. Nanotube Circuit. The electrodes have a width of $10 \mu \mathrm{m}$ a) Optical microscope image of a nanotube transistor. b) AFM image of a CNT transistor showing CNTs forming complete circuits. c) AFM of an individual CNT. Height range is 0 (black) to $2.5 \mathrm{~nm}$ and above (white).

\subsection{Fabrication of Individual CNT circuits}

The CNT transistors used in the current experiments are created on silicon wafers that are p-doped and have a $300 \mathrm{~nm}$ top-surface oxide (Silicon Quest). An example of a finished device is shown in Figure 3. Before nanotube growth, alignment markers are patterned onto the chip using photolithography as shown in Figure 4a-c. The photoresist (S1813 from Microchem) is spun to nominal thickness $1.3 \mu \mathrm{m}$ and patterned using a contact aligner (Karl Suss). After development, the photoresist is hardbaked $(5 \mathrm{~min}$ at $115 \mathrm{C}$ ) to prepare the resist for an oxide etch. Alignment markers are etched into the $\mathrm{SiO}_{2}$ using buffered $\mathrm{HF}$ (20:1 buffer to $\mathrm{HF}$ ratio, total etch depth $100 \mathrm{~nm}$ ).

a)

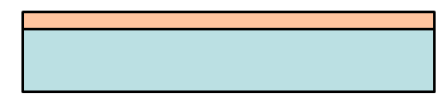

d)

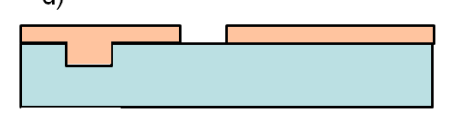

g)

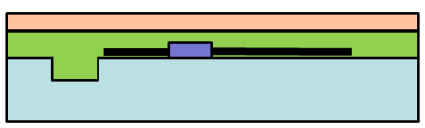

b)

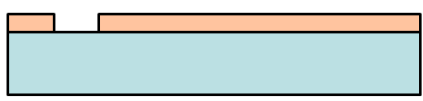

e)

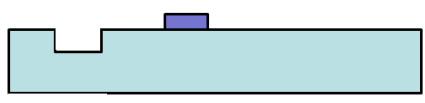

h)

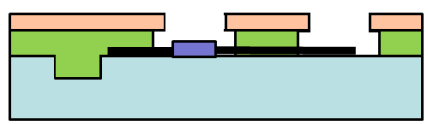

c)

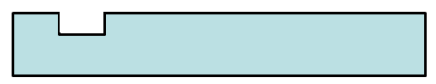

f)

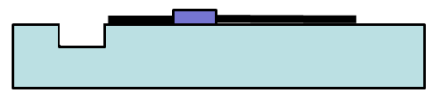

i)

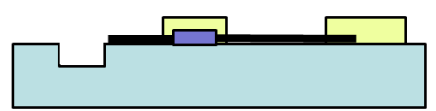

Figure 4. The biosensor device fabrication process. a) Photoresist is spun onto a silicon wafer. b) Photoresist is soft-baked, exposed, and developed, and hard-baked. c) The oxide is etched and the photoresist is removed. d) The wafer is patterned with photoresist and preparing for catalyst deposition. e) Catalyst is deposited onto the wafer and the photoresist is removed. f) Nanotubes are grown by CVD. g) Bilayer photoresist is spun onto the wafer. h) The photoresist is patterned and ready for metal evaporation. i) $\mathrm{Cr} / \mathrm{Au}$ is thermally evaporated onto the wafer.

To selectively deposit catalyst on the chip (Figure 4d-e), a second photolithography step is performed. A wafer with patterned photoresist (S1813) is then exposed to catalyst by either drop casting a catalyst solution, ${ }^{9}$ spinning on a catalyst 
solution, ${ }^{12}$ or electron-beam evaporation of a thin metal layer. ${ }^{10}$ All three catalysts produce good results and differences between the methods are currently under investigation. The drop-cast catalyst solution is alumina supported CNT catalyst dissolved in DI water and has $1.3 \mathrm{mg} / \mathrm{ml}$ iron nitrate $\mathrm{Fe}\left(\mathrm{NO}_{3}\right)_{3}, 0.3 \mathrm{mg} / \mathrm{ml}$ Molybdenum (VI) dioxide bis(acetylacetonate), and $1 \mathrm{mg} / \mathrm{ml}$ of nano-powdered alumina $\left(\mathrm{Al}_{2} \mathrm{O}_{3}\right.$ from Degussa). The spun-on catalyst solution has $1 \mathrm{mg} / \mathrm{ml}$ PVP (polyvinylpyrrrolidone MW $360 \mathrm{kDa}$ ) (Sigma Aldrich), $1 \mathrm{mM}$ iron nitrate (Sigma-Aldrich), and $0.5 \mathrm{mM}$ Molybdenum (VI) dioxide bis(acetyl-acetonate) (Strem Chemicals). The PVP increases the viscosity of the solution to give a uniform spin coat. Electron beam evaporated catalyst is pure iron and $0.2 \mathrm{~nm}$ thick. Once the catalyst has been deposited, the photoresist is removed by rinsing sequentially with acetone, isopropyl alcohol, and DI water. Small rectangles of catalyst (6x10um) are left behind.

Catalyzed CVD growth of nanotubes is performed in a one-inch tube furnace using growth parameters based on an existing recipe. ${ }^{13}$ Wafers are diced into $2 \times 2 \mathrm{~cm}$ sections (small enough to fit in the one-inch tube furnace) and annealed in open air at $750{ }^{\circ} \mathrm{C}$ for $5 \mathrm{~min}$. The furnace is then sealed, flushed with argon, and annealed with $\mathrm{H}_{2}$ at $0.45 \mathrm{SLM}$ for 10 min to remove oxygen from the metal catalyst. The furnace is flushed again with argon and ramped up to the nanotube growth stage at $900 \mathrm{C}$. The gases during growth are $0.45 \mathrm{SLM} \mathrm{H}_{2}, 0.15 \mathrm{SLM}$ Ar bubbled through ethanol, and $0.3 \mathrm{SLM}$ Ar bubbled through methanol. The growth time is $15 \mathrm{~min}$. The furnace is then cooled under an Ar flow. This growth technique produces CNTs with a range of diameters, from 1-3 nm.

The last step of circuit fabrication is the deposition of metal electrodes (Figure 4g-i). The chip is coated with a bi-layer photoresist (LOR3B and S1813 from Microchem). The under-layer (LOR3B) ensures an undercut profile which aids in metal lift off. After patterning the photoresist, the chip is placed in a thermal evaporator. The electrodes metals are $\mathrm{Cr} / \mathrm{Au}(5 \mathrm{~nm} / 30 \mathrm{~nm})$ with the $\mathrm{Cr}$ layer promoting adhesion of the Au to the oxide surface. The evaporation rate is $1 \mathrm{~A} / \mathrm{s}$ and residual gas pressure is $7 \times 10^{-6}$ Torr or lower. After the metal deposition, photoresist and excess metal is lifted off by soaking in remover (Remover PG from Microchem) at $60^{\circ} \mathrm{C}$ for 1 hour. The chip is rinsed sequentially with acetone, isopropyl alcohol, and DI water before drying.

The photoresist used during fabrication leaves organic residues on the circuit. An effective way to clean the circuit before a biosensing experiment is to anneal for 10 minutes at $400^{\circ} \mathrm{C}$ in an $\mathrm{Ar}$ atmosphere (1 SLM Ar in a one-inch tube furnace) ${ }^{14}$ Previous authors have noted the importance of this step before selective functionalizing of CNT can be achieved (section 4). ${ }^{15}$ The annealing step affects the device properties, often increasing the contact resistance between the metal and the CNT. This may be due to oxidation of the Cr sticking layer and is currently under investigation.
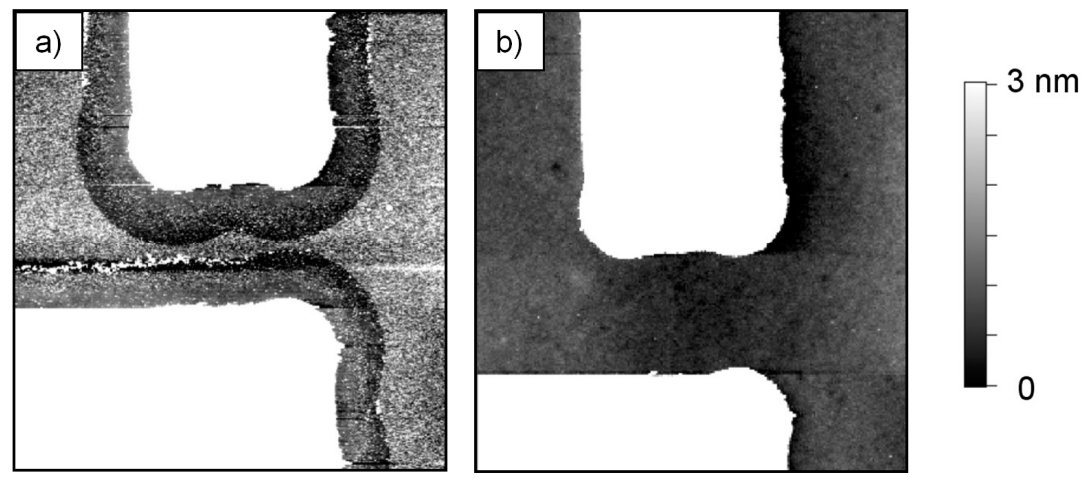

Figure 5. AFM images showing the effectiveness of surface cleaning. a) Metal electrodes (white) and the oxide surface directly after lift off. The electrodes are $10 \mu \mathrm{m}$ wide. b) The same area after a 10 minute anneal in $1 \mathrm{SLM} \mathrm{Ar}$ at $400^{\circ} \mathrm{C}$. The height scales for both images are identical. There is no CNT in this test circuit. 


\subsection{Fluid delivery to the biosensor chip}

Microfluidic channels made from polydimethylsiloxane (PDMS) are a well established method to deliver small volumes of solution to the active area of a biosensor. ${ }^{16}$ PDMS channels can form temporary or permanent seals to silicon wafers and have potential for "lab on a chip" applications.
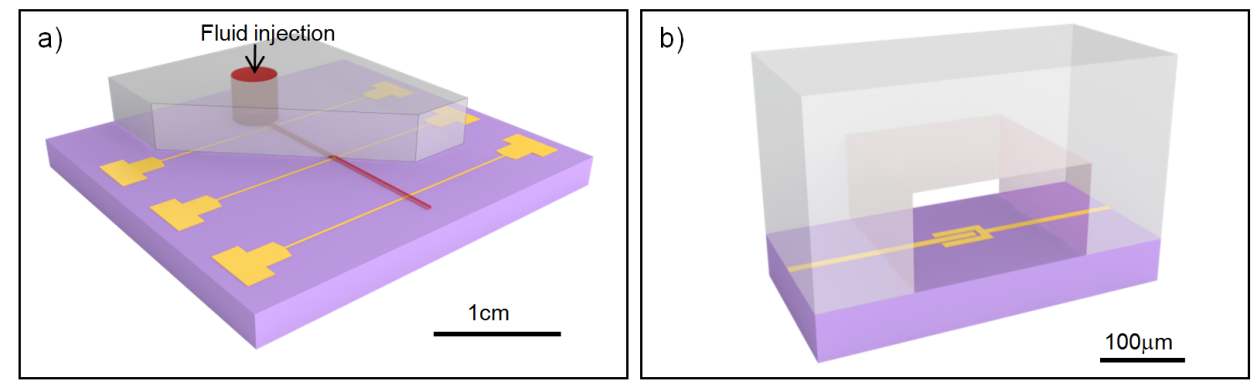

Figure 6. a) Cross section of a PDMS channel (gray) on our CNT FET biosensor chip (purple). Fluid is colored red for clarity. b) Magnified image showing the gap between electrodes and the width of the microfluidic channel.

The mold for creating a PDMS flow channel is made from $100 \mu \mathrm{m}$ thick photoresist (SU8 3050 from Microchem). The photoresist mold is patterned in a contact aligner and then developed following the manufacturer's guidelines. PDMS is mixed (10:1 ratio of resin to hardener) and then poured on top of the photoresist mold. The PDMS is cured overnight at room temperature and then removed from the mold. The resulting flow channels are $100 \mu \mathrm{m}$ tall and $200 \mu \mathrm{m}$ wide.

The PDMS channel is pressed onto a CNT FET biosensor chip to form a water-tight seal with the oxide surface. We use a home-built acrylic fixture to clamp the PDMS in place. The fixture connects to inlet and outlet tubing (Teflon tubing, 0.009in inner diameter, Upchurch Scientific) and holds a $\mathrm{Ag} / \mathrm{AgCl}$ reference electrode (model MF-2078, BAS) in contact with the solution in the channel. On the nanotube chip there is a gold working electrode which is used to gate the solution. The reference electrode is used to measure $V_{\mathrm{g}}$. For small gating voltages (a small voltage produces under $\ln \mathrm{A}$ of faradaic current) the reference electrode can be used as both the working electrode and reference electrode with minimal error. ${ }^{17}$

\subsection{Sensor response time and calibration}

When delivering solution to the sensor, the lead volume must be flushed. The lead volume is the space in between the new solution and the sensor. A pH detection experiment was designed to find the lag time to reach fully-developed flow after solutions are switched (see Fig 7).
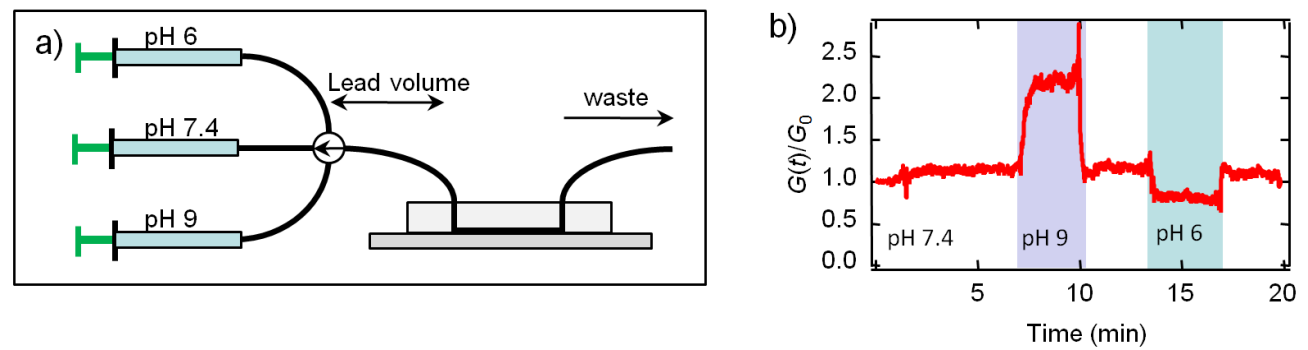

Figure 7. $\mathrm{pH}$ calibration on a nanotube circuit. a) Diagram of our microfluidic system showing 3 syringes with solutions of PBS at differing $\mathrm{pH}$. A microfluidic hub (Upchurch Scientific, dead volume $12 \mu \mathrm{l}$ ) switches between different syringes. The lead volume of tubing is $6 \mu \mathrm{l}(15 \mathrm{~cm}$ length, $225 \mu \mathrm{m}$ inner diameter) and lead volume in the PDMS is $6 \mu 1$. b) Plot of nanotube conductance as the $\mathrm{pH}$ of PBS buffer is changed. A constant flow rate of $10 \mathrm{ml} / \mathrm{hr}$ is controlled by a syringe pump. 
Figure 7 shows that the $\mathrm{pH}$ sensing signal takes 30 - $60 \mathrm{sec}$ to develop when using a pressure-driven flow rate of 10 $\mathrm{ml} /$ hour. For slower flow rates $(1 \mathrm{ml} /$ hour $)$ the signal settles in $100-200 \mathrm{sec}$. This lag time is also a function of the length and cross-section of piping in the microfluidics and the diffusion constant of the molecule being delivered to the sensor. Fast flow rates are desirable for minimizing the lead time, however, high flow rates use more sample volume and potentially create systematic sensing errors. For example, high flow rates are known to change the electrostatic potential at the sensor surface, thereby interfering with the sensing of charged proteins. ${ }^{18}$

The $\mathrm{pH}$ sensing experiment gives a lower limit of the lag-time for proteins to begin binding to a biosensor. Protons diffuse rapidly across flow lines and will reach the sensor surface faster than proteins. A spherical protein with a mass of $50 \mathrm{kDa}$ has $D \sim 10^{-10} \mathrm{~m}^{2} \mathrm{~s}^{-1}$ (diffusion constants can easily be estimated from the Einstein relation $D=k_{\mathrm{B}} T / 6 \pi \eta r$, where $k_{\mathrm{B}}$ is the Boltzman constant, $T$ is temperature, $\eta$ is the viscosity of water, and $r$ is the protein radius which we estimate by assuming spherical geometry and protein density $1.2 \times 10^{3} \mathrm{~kg} / \mathrm{m}^{3}$ ). Thus, the typical diffusion constant of a protein in solution is 100 times less than the diffusion constant of a proton in solution.

Figure 8 shows the effective change in gate voltage when $\mathrm{pH}$ is changed $\Delta V_{\mathrm{g}}$. The function $G\left(V_{\mathrm{g}}\right)$ shown in Fig. $8 \mathrm{a}$ was used to convert $G(t)$ data from Figure 7 into $\Delta V_{\mathrm{g}}(t)$. Changing the $\mathrm{pH}$ of the solution causes changes in the charge density at the $\mathrm{SiO}_{2}$-water interface. When $\mathrm{pH}$ is increased to 9 , the surface loses protons and $\Delta V_{\mathrm{g}}$ is negative. When $\mathrm{pH}$ is decreased, the surface is protonated and $\Delta V_{\mathrm{g}}$ is positive. These measurements are a useful calibration of the performance of the charge-sensitive biosensor. The theoretical maximum change in surface potential for $\mathrm{SiO}_{2}$ is $60 \mathrm{mV} / \mathrm{pH} .{ }^{19}$ Figure $8 \mathrm{~b}$ shows a change of roughly $40 \mathrm{mV} / \mathrm{pH}$, and we have observed a range of sensitivities from $10-50 \mathrm{mV} / \mathrm{pH}$. Variability may be due to organic residues on the $\mathrm{SiO}_{2}$ surface or variability in CNT diameters.

a)

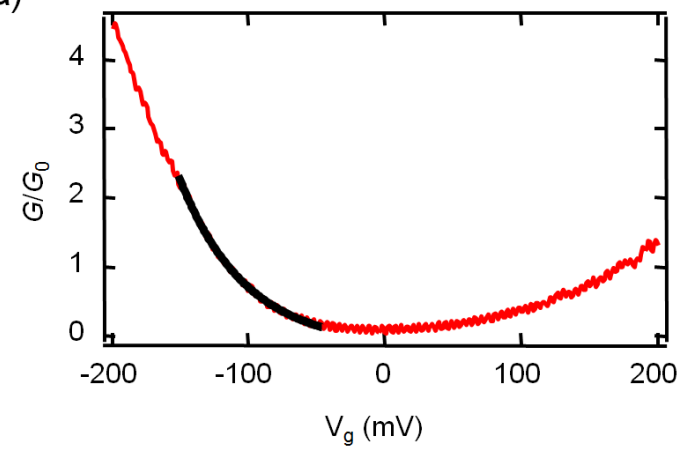

b)

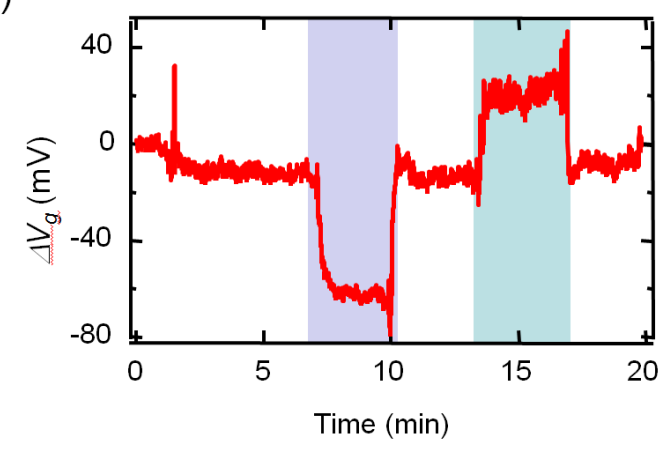

Figure 8 a) Transistor curve of the nanotube circuit from Figure 7, the black line is fit to the curve and is used to extrapolate the effective shift in gate voltage $\Delta V_{\mathrm{g}}$ that is caused by changes in $\mathrm{pH}$. The bulk solution potential $V_{\mathrm{g}}$ is set to $-115 \mathrm{mV}$ during the experiment. b) Plot of the shift in potential $\Delta V_{\mathrm{g}}$. The shift is zero at $\mathrm{pH} 7.4$ and changes by $46 \mathrm{mV}$ when changing to $\mathrm{pH} 9$, and changes by $36 \mathrm{mV}$ when changing to $\mathrm{pH} 6$.

Temperature fluctuations can be a source of systematic error for nanoFET biosensor experiments. Changes in temperature can be introduced when warmer or cooler liquids are introduced to the microfluidic system. The most sensitive operating point of CNT-FET biosensor is the subthreshold region ${ }^{8}$ where conductance is given by, ${ }^{20}$

$$
G=G_{o} e^{-e\left(V_{g}-V_{t h}\right) / k_{B} T},
$$

where e is the charge of an electron and $V_{\text {th }}$ is the threshold gate voltage for the transistor to turn on. Equation 1 shows that device conductance depends exponentially on temperature. 
Figure 9 illustrates how temperature sensitivity depends on $V_{\mathrm{g}}$. At a typically operating point $\left(V_{\mathrm{g}}-V_{\mathrm{th}}=200 \mathrm{mV}\right)$ conductance can swing by over $2 \%$ for a temperature change of $1{ }^{\circ} \mathrm{C}$. The issue of temperature control can be addressed by actively correcting temperature fluctuations using an on-chip temperature sensor and heater. An alternative, low-tech approach is to let all solutions equilibrate to room temperature before beginning a measurement.

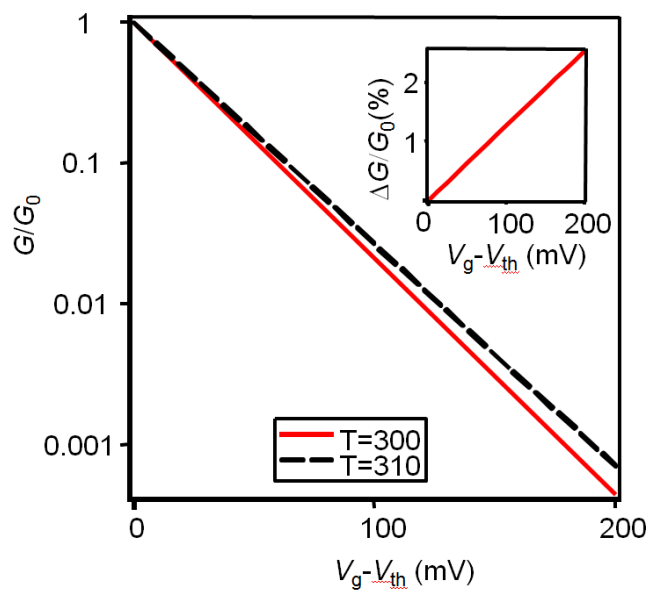

Figure 9. Temperature dependence of a CNT FET's conductance as a function of the difference between gate voltage $V_{\mathrm{g}}$ and the threshold voltage $V_{\text {th. }}$. The insert shows the percent change in conductance for a temperature difference of $1^{\circ} \mathrm{C}$.

\section{FUNCTIONALIZATION OF NANOTUBES}

In order to make a useful biological sensor, the surface of the sensor must bind the specific biomolecule of interest while rejecting other biomolecules. Specific binding of ss-dna can be achieved by using the complementary ss-dna. Specific binding of proteins can be achieved using antibodies or aptamers, as described below.

To functionalize the carbon nanotube we have followed a protocol developed by Chen et al. ${ }^{21}$ Clean CNTs are incubated in a $1 \mathrm{mM}$ solution of 1-pyrenebutanoic acid succinimidyl ester (PBASE, sold by Molecular Probes) in dimethylformamide (DMF). The incubation time is 5 hours. PBASE is chosen due to pyrene's ability to pi-stack on the CNT surface. The succinimidyl ester provides a handle for biomolecular conjugation. Succinimidyl ester is degraded by water and light, therefore, the DMF is dried of water using molecular sieves, and the solution is protected from light. After incubation, the chip is rinsed in dry DMF and then dried in nitrogen. A drop of $1 \mathrm{mM}$ ferritin in PBS was placed on the PBASE-functionalized CNTs and incubated for $3 \mathrm{hrs}$. Ferritin was chosen because it is easy to image by atomic force microscopy and the primary amines on ferritin will conjugate to the succinimidyl ester. The resulting conjugation of ferritin to CNTs is shown in Figure 10. The scheme can easily be adapted to conjugate antibodies or aptamers to the CNT instead of ferritin. 

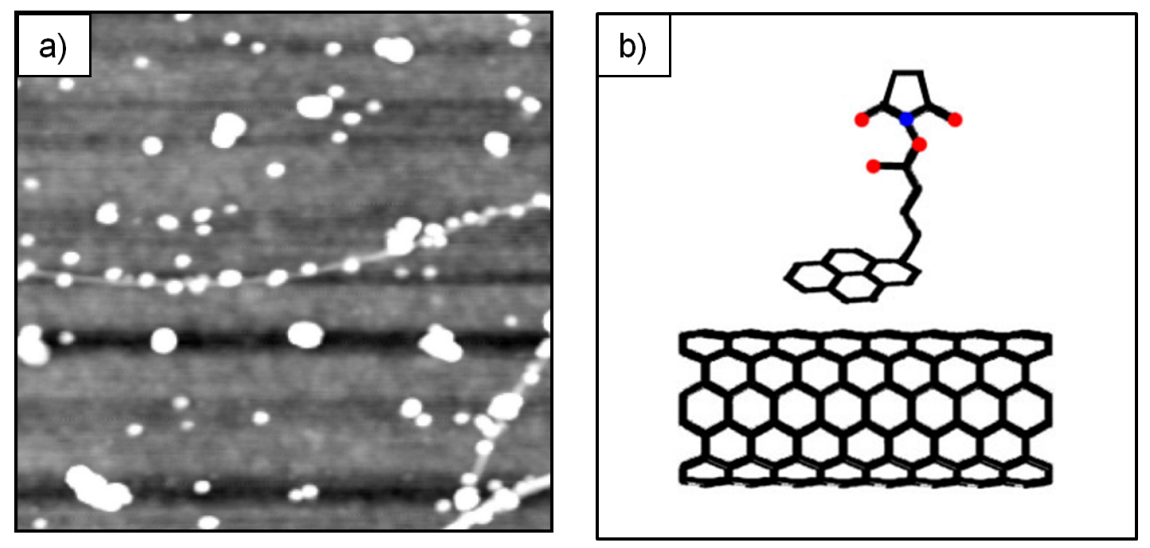

Figure 10. Conjugation of ferritin to CNTs. a) AFM image $(1 \mu \mathrm{mx} 1 \mu \mathrm{m})$ of CNTs (white lines) that have been treated with PBASE and then ferritin (white dots). Some ferritin complexes stick specifically to the CNT, while others stick nonspecifically to the silicon oxide surface. b) Schematic of the specific binding scheme. PBASE sticks to the side walls of the CNT by $\pi$ stacking.

\section{Electrostatic screening in solution}

When a charged protein adsorbs to the surface of a CNT biosensor, the electrostatic coupling between the protein and the CNT depends on separation distance and counter-ion screening. Counter ion screening is characterized by the Debye screening length

$$
\lambda_{D}=\frac{1 \mathrm{~nm}}{\sqrt{c / 93 \mathrm{mM}}}
$$

where $c$ is the monovalent salt concentration. The CNT biosensor response will decay exponentially with separation distance. The exponential decay constant is $\lambda_{\mathrm{D}}$. To simulate biological conditions in our experiments we use phosphate buffered saline (PBS) with $\lambda_{\mathrm{D}} \sim 1 \mathrm{~nm}\left(137 \mathrm{mM} \mathrm{NaCl}\right.$ and $10 \mathrm{mM} \mathrm{Na}_{3}\left(\mathrm{PO}_{4}\right)$, therefore separation distances must be $\sim 1$ $n m$ for optimal sensing.

Typical functionalization schemes utilize antibodies to specifically catch target biomolecules from solution. The antibody acts as 10-15 nm spacer between the CNT and the target biomolecule. Therefore, biosensing in biological conditions $\left(\lambda_{\mathrm{D}} \sim 1 \mathrm{~nm}\right)$ is not practical. Some authors have solved this issue by using low salt buffers. ${ }^{22,23}$ Besides adding an additional sample preparation step, the use of low-salt buffers may reduce the affinity of antibody-antigen binding. Low salt concentrations can also lead to weak $\mathrm{pH}$ buffering (PBS is held at a stable $\mathrm{pH}$ by the buffering properties of phosphate ions). As shown in Fig. 7, fluctuations in $\mathrm{pH}$ will lead to large signals.

An alternative approach to the issue of electrostatic screening is to reducing the size of the functionalization molecule on the biosensor surface. Aptamers are a few nanometers in size and are a promising alternative to antibodies. ${ }^{24}$

\section{ANALYZING SENSING DATA}

Figure 11 shows two example sensing experiments that use biological molecules of different charge: polylysine (one positive charge per monomer at $\mathrm{pH}$ 7.4) and ss-dna (one negative charge per monomer at $\mathrm{pH}$ 7.4). From Figure 11 we see that polylysine binding is equivalent to a shift in gate voltage $\Delta V_{\mathrm{g}}=+55 \mathrm{mV}$. In other experiments we have observed a range $30-90 \mathrm{mV}$. From Figure 11 we see that ss-dna binding is equivalent to $\Delta V_{\mathrm{g}}=-45 \mathrm{mV}$. 
We attribute sign of $\Delta V_{\mathrm{g}}$ to the polarity of the biological molecules. Differences in the magnitude of $\Delta V_{\mathrm{g}}$ are likely related to differences in binding geometry. Fluorescence imaging (not shown) reveals that polylysine binds to all bare silicon oxide surfaces. This binding is driven by the Coulomb interaction between negatively charged $\mathrm{SiO} 2\left(-2.5 \mu \mathrm{C} / \mathrm{m}^{2}\right.$ ${ }^{25}$ ) and positively charged polylysine. In contrast, previous studies of ss-dna show that the ss-dna only binds to the CNT and not the $\mathrm{SiO}_{2}{ }^{26}$

a)

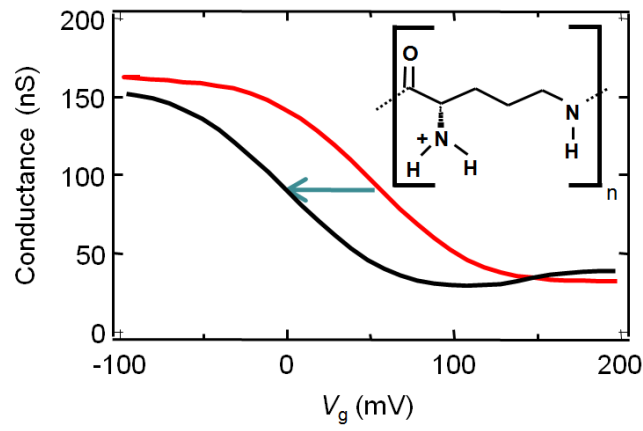

b)

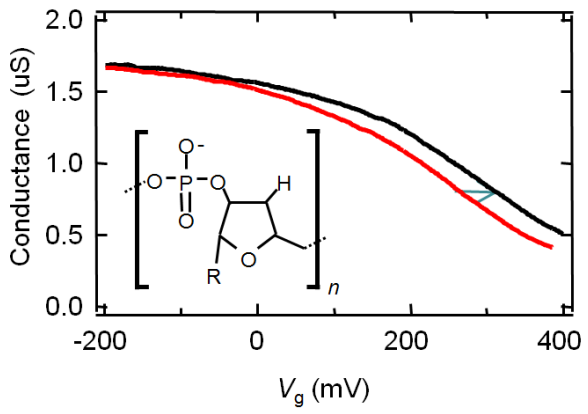

Figure 11. Biosensor signals depend on the charge of the biomolecule. a) Transistor curves measured before (red) and after (black) polylysine binding. The curve shifts $55 \mathrm{mV}$ to the left, equivalent to $\Delta V_{\mathrm{g}}=+55 \mathrm{mV}$. b) Transistor curves measured before (red) and after (black) the binding of ss-dna. The curve shifts by about $45 \mathrm{mV}$ to the right.

To compare $\Delta V_{\mathrm{g}}$ due to polylysine binding to theoretical estimates we use the Gouy-Chapman Stern model. In this model, a layer of cations are bound to the negatively charged $\mathrm{SiO}_{2}$ surface (this Stern layer is $<0.5 \mathrm{~nm}$ thick). A diffuse layer of additional screening ions extends into the solution. The diffuse layer can maintain a maximum potential difference of roughly $4 k_{\mathrm{B}} T$, or $100 \mathrm{mV} .^{27}$ Our CNTs range in diameter from $1-2 \mathrm{~nm}$, therefore the majority of the CNT sits in the diffuse layer where $\Delta V_{\mathrm{g}}$ is limited to $100 \mathrm{mV}$. This is consistent with our experiments where the highest measured shift for polylysine was $90 \mathrm{mV}$.

Figure $2 \mathrm{~b}$ shows the real time binding of polylysine to a CNT sensor. The detection speed in this experiment is limited by the rate of protein binding which we can estimate using mass-transport theory as follows. In a simple model of protein flux we have, ${ }^{28}$

$$
\begin{aligned}
& \delta=\left(\frac{D L}{\dot{\gamma}}\right)^{1 / 3}, \\
& \text { Flux }=\frac{D C_{o}}{\delta},
\end{aligned}
$$

where $\delta$ is the depletion length, $\gamma$ is the shear rate, $L$ is the length of the binding region, and $C_{\mathrm{o}}$ is the original concentration proteins in solution. For the experiment shown in Fig. $2 \mathrm{~b}, L=1.4 \mathrm{~mm}, \gamma=27 \mathrm{~s}^{-1}, C_{\mathrm{o}}=90 \mathrm{nM}$, and $D=4 \mathrm{x}$ $10^{-13} \mathrm{~m}^{2} \mathrm{~s}^{-1} \cdot{ }^{29}$ Using equations 3 and 4 we can calculate a flux of $8 \times 10^{12} \mathrm{~m}^{-2} \mathrm{~s}^{-1}$.

To compare this above theoretical prediction to observed protein flux, we consider the time for the signal to saturate. Figure $2 \mathrm{~b}$ shows a clear signal saturation after approximately 1.5 minutes. This saturation occurs when accumulated polylysine neutralizes the charged $\mathrm{SiO}_{2}$ surface (the bare charge of $\mathrm{SiO}_{2}$ is $-2.5 \mu \mathrm{C} / \mathrm{cm}^{2}$ or roughly $-e$ per $6 \mathrm{~nm}^{2}$, where $e$ is the charge of the electron) ${ }^{25}$. Since each polylysine molecule carries a charge of $+800 e$, the predicted flux of $8 \times 10^{12} \mathrm{~m}^{-}$ ${ }^{2} \mathrm{~s}^{-1}$ will lead to charge neutrality after $26 \mathrm{~s}$, faster than the time for fully developed flow (Fig. 7). Therefore, the observed 1.5 min saturation time is consistent with theoretical predictions for mass transport. 
Based on this simple mass-transport theory, and the signal-to-noise ratio in our CNT FET biosensors, we can estimate the lowest concentration of polylysine that is compatible with rapid detection. When polylysine binding reaches $20 \%$ saturation the signal is well above the noise level (Figure 2b). We arbitrarily define rapid detection as 5 minutes. Using other sensor parameters described above, we conclude that the lower limit for rapid detection is $8 \mathrm{nM}$. We are currently seeking ways to decrease this lower limit, to access the picomolar concentrations that are most relevant for medical diagnostics.

Acknowledgment: This work was funded by the Army Research Labs via the Oregon Nanoscience and Microtechnology Institute (ONAMI).

\section{REFERENCES}

[1]Homola, J. "Present and future of surface plasmon resonance biosensors" Analytical and Bioanalytical Chemistry 377, 528 (2003).

[2]Yan, R.,Mestas, S.,Yuan, G.,Safaisini, R.,Dandy, D. Lear, K. "Label-free silicon photonic biosensor system with integrated detector array" Lab on a Chip 9, 2163 (2009).

[3]Daniels, Jonathan S. Pourmand, N. "Label-Free Impedance Biosensors: Opportunities and Challenges"

Electroanalysis 19, 1239 (2007).

[4]Waggoner, P. S. Craighead, H. G. "Micro- and nanomechanical sensors for environmental, chemical, and biological detection" Lab on a Chip 7, 1238 (2007).

[5]Patolsky, F.,Zheng, G. Lieber, C. M. "Nanowire-Based Biosensors" Analytical Chemistry 78, 4260 (2006).

[6]Allen, B. L.,Kichambare, P. D. Star, A. "Carbon Nanotube Field-Effect-Transistor-Based Biosensors" Advanced Materials 19, 1439 (2007).

[7]Heath, J. R.,Davis, M. E. Hood, L. "Nanomedicine-Revolutionizing the Fight against Cancer" Scientific American, (2009).

[8]Heller, I.,Janssens, A. M.,Mannik, J.,Minot, E. D.,Lemay, S. G. Dekker, C. "Identifying the Mechanism of Biosensing with Carbon Nanotube Transistors" Nano Letters 8, 591 (2007).

[9]Kong, J.,Soh, H. T.,Cassell, A. M.,Quate, C. F. Dai, H. "Synthesis of individual single-walled carbon nanotubes on patterned silicon wafers" Nature 395, 878 (1998).

[10]Kang, S. J.,Kocabas, C.,Ozel, T.,Shim, M.,Pimparkar, N.,Alam, M. A.,Rotkin, S. V. Rogers, J. A. "High-

performance electronics using dense, perfectly aligned arrays of single-walled carbon nanotubes" Nat Nano 2, 230 (2007).

[11]Wang, C.,Zhang, J.,Ryu, K.,Badmaev, A.,De Arco, L. G. Zhou, C. "Wafer-Scale Fabrication of Separated Carbon Nanotube Thin-Film Transistors for Display Applications" Nano Letters 9, 4285 (2009).

[12]Ding, L.,Yuan, D. Liu, J. "Growth of High-Density Parallel Arrays of Long Single-Walled Carbon Nanotubes on Quartz Substrates" Journal of the American Chemical Society 130, 5428 (2008).

[13]Ding, L.,Tselev, A.,Wang, J.,Yuan, D.,Chu, H.,McNicholas, T. P.,Li, Y. Liu, J. "Selective Growth of Well-Aligned Semiconducting Single-Walled Carbon Nanotubes" Nano Letters 9, 800 (2009).

[14]Ishigami, M.,Chen, J. H.,Cullen, W. G.,Fuhrer, M. S. Williams, E. D. "Atomic Structure of Graphene on SiO2" Nano Letters 7, 1643 (2007).

[15]Zhu, J.,Brink, M. McEuen, P. L. "Single-Electron Force Readout of Nanoparticle Electrometers Attached to Carbon Nanotubes" Nano Letters 8, 2399 (2008).

[16]Duffy, D. C.,McDonald, J. C.,Schueller, O. J. A. Whitesides, G. M. "Rapid Prototyping of Microfluidic Systems in Poly(dimethylsiloxane)" Analytical Chemistry 70, 4974 (1998).

[17]Minot, E.,Janssens, A.,Heller, I.,Heering, H.,Dekker, C. Lemay, S. "Carbon nanotube biosensors: The critical role of the reference electrode" Applied Physics Letters 91, 093507 (2007).

[18]Bourlon, B.,Wong, J.,Miko, C.,Forro, L. Bockrath, M. "A nanoscale probe for fluidic and ionic transport" Nat Nano 2, 104 (2007). 
[19]Hal van, R. E. G.,Eijkel, J. C. T. Bergveld, P. "A novel description of ISFET sensitivity with the buffer capacity and double-layer capacitance as key parameters" Sensors and Actuators B: Chemical 24-25, 201 (1995).

[20]Rosenblatt, S.,Yaish, Y.,Park, J.,Gore, J.,Sazonova, V. McEuen, P. L. "High Performance Electrolyte Gated Carbon Nanotube Transistors" Nano Letters 2, 869 (2002).

[21]Chen, R. J.,Zhang, Y.,Wang, D. Dai, H. "Noncovalent Sidewall Functionalization of Single-Walled Carbon Nanotubes for Protein Immobilization" Journal of the American Chemical Society 123, 3838 (2001).

[22]Stern, E.,Wagner, R.,Sigworth, F. J.,Breaker, R.,Fahmy, T. M. Reed, M. A. "Importance of the Debye Screening Length on Nanowire Field Effect Transistor Sensors" Nano Letters 7, 3405 (2007).

[23]Zheng, G.,Patolsky, F.,Cui, Y.,Wang, W. U. Lieber, C. M. "Multiplexed electrical detection of cancer markers with nanowire sensor arrays" Nat Biotech 23, 1294 (2005).

[24]Maehashi, K.,Katsura, T.,Kerman, K.,Takamura, Y.,Matsumoto, K. Tamiya, E. "Label-Free Protein Biosensor Based on Aptamer-Modified Carbon Nanotube Field-Effect Transistors" Analytical Chemistry 79, 782 (2006).

[25]Artyukhin, A. B.,Stadermann, M.,Friddle, R. W.,Stroeve, P.,Bakajin, O. Noy, A. "Controlled Electrostatic Gating of Carbon Nanotube FET Devices" Nano Letters 6, 2080 (2006).

[26]Star, A.,Tu, E.,Niemann, J.,Gabriel, J.,Joiner, C. Valcke, C. "Label-free detection of DNA hybridization using carbon nanotube network field-effect transistors" Proceedings of the National Academy of Sciences of the United States of America 103, 921 (2006).

[27]Bard, A. J. Faulkner, L., [ELECTROCHEMICAL METHODS Fundamentals and Apllications ], John Wiley\& Sons, (2001).

[28]Squires, T. M.,Messinger, R. J. Manalis, S. R. "Making it stick: convection, reaction and diffusion in surface-based biosensors" Nat Biotech 26, 417 (2008).

[29]Rodriguez-Maldonado, L.,Fernandez-Nieves, A. Fernández-Barbero, A. "Dynamic light scattering from high molecular weight poly-1-lysine molecules" Colloids and Surfaces A: Physicochemical and Engineering Aspects 270, 335 (2005). 